Relations industrielles

Industrial Relations

\title{
Faut-il prévenir le patron
}

\section{Omer Genest}

Volume 2, numéro 6, février 1947

URI : https://id.erudit.org/iderudit/1023865ar

DOI : https://doi.org/10.7202/1023865ar

Aller au sommaire du numéro

\section{Éditeur(s)}

Département des relations industrielles de l'Université Laval

ISSN

0034-379X (imprimé)

1703-8138 (numérique)

Découvrir la revue

Citer cet article

Genest, O. (1947). Faut-il prévenir le patron. Relations industrielles / Industrial Relations, 2(6), 7-8. https://doi.org/10.7202/1023865ar

Tous droits réservés @ Département des relations industrielles de l’Université Laval, 1947
Ce document est protégé par la loi sur le droit d'auteur. L’utilisation des services d'Érudit (y compris la reproduction) est assujettie à sa politique d'utilisation que vous pouvez consulter en ligne.

https://apropos.erudit.org/fr/usagers/politique-dutilisation/ 


\section{PRACTICAL CASES}

Can an employer validly impose on latecomers amongst his employees a fine equivalent to one quarter of an hour's pay for each five minutes' delay in coming to work?

\section{Could you also tell me by what criterion the validity} of a contractual provision may be ascertained?

The validity of a provision may be established by a criterion which is to be found in the two following sections of the Civil Code:

A-Section 13: "No one can, by private agreement, validly contravene the laws of public order and good morals".

B-Section 14: "Prohibitive laws import nullity although such nullity be not therein expressed."

Public order is that upon the maintainance of which the existence of society depends. Indeed, the maintainance of public order is the very purpose of the enactment of Constitutional, Administrative and Criminal law, the laws concerning family rights, the status or capacity of persons, the prevention of fraud, the protection of individuals, in short, Public Law as a whole.

The term good morals means monals consistent with Christian ethics.

Prohibitive laws imply a prohibition; therefore, in virtue of such laws, certain things are forbidden. Now, section 14 of the Civil Code deals with the consequenses of the violation of such laws by decreeing that anything which contravenes their provisions is null, even though such nullity be not explicitly mentioned in their wording.

From the foregoing principles it follows that the criterion in question comprises three essential items which must be equally taken into account in appraising a given provision.

Is such provision contrary to public order? Is it inconsistent with good morals? Is it contrary to a prohibitive law? Obviously, if "no" is the answer in each case, the provision in question is valid. On the other hand, if "yes" is the answer to even just one of these questions, the provision being examined must be deemed illegal.

In this particular case, it is a question of knowing whether or not an emyloyer has the right to penalize those amongst his employees who come in late to work by making them lose a certain amount of time.

In fact, it seems to us that such a penalty is by no means contrary to public order, inasmuch as its application does not involve a departure from the wage rates fixed by Decree or prescribed by the Ordinances of the Minimum Wage Commission. It is merely a disciplinary measure intended to ensure punctuality. Besides, who would deny that in a large industry, where rationalization has set up work in series, an employer would sustain a considerable loss through an employee's mere delay in coming to work. Hundreds of operations could thus be impaired...

Would such a penalty be inconsistent with good morals? No! unless it were imposed with such exaggeration that it would cease to be a mere disciplinary measure and become equival:nt to robbery, i.e. a means of exploiting the worker.

However, we would advise the employer not to "apply" such penalties too mercilessly nor too generally so as to avoid the possibility of injustice in certain particular cases. Sufficiently justified delays in coming to work should be generously overlooked.

Finally, to the best of our knowledge, there exists no law forbidding employers to penalize latecomers. Consequently, since we have given a negative answer to each of our three-questions, we may infer that the manner in which this employer treats the latecomers amongst his employees is by no means unlawful as long as he applies such penalties within reasonable limits and does not profit by them to violate the provisions of a Decree or depart from the minimum wage rates fixed by the Ordinances. Nevertheless, as concerns sound industrial relations, could it be said that such a disciplinary measure is as wise as effective? It would be preferable if the fines collected were placed in a fund specially intended to promote the organizing of social services within the enterprise.

Donat QUIMPER

\section{FAUT-IL PREVENIR LE PATRON}

(Suite à la page 8)

Que leur union va changer cette situation, le patron le sent bien et il est normal que ses premières impressions ne soient guère sympathiques. Mais le patron intelligent réfléchit et se rend vite compte qu'il gagnera à ce changement. Il assurera à son entreprise la collaboration organisée de son personnel, qui, secouant l'état de sujétion où il était, accroît sa valeur humaine et ses possibilités de rendement. L'expérience ne tardera pas à lui montrer que les syndiqués les plus fervents forment l'élite de sa main-d'oeuvre. Cependant, la première réaction du patron est toujours à craindre car elle peut pousser à des attitudes hostiles qui menacent la vie du syndicat naissant.

Quant aux demandes extravagantes, le patron a toujours la liberté de les discuter et de les refuser. Mais en accédant à une juste requête de ses employés, loin de se diminuer, il pose un geste qui sera beaucoup plus apprécié que toute libéralité teintée d'un certain paternalisme orgueilleux: "Je donne parce que je veux, et non parce qu'ils me demandent ».

Concluons: dans la quasi généralité des cas, il est normal que les ouvriers ne préviennent pas leur patron de leur intention de s'unir. En se groupant ils ne font qu'user de leur liberté d'association que le droit naturel et la loi leur garantissent. (Loi des relations ouvrières, art. 3,20,21,22). L'employeur qui n'a pas été averti peut ne •pas aimer ce silence, mais il a tort de croire que ses travailleurs ou les organisateurs d'unions lui jouent dans le dos. Il lui est beaucoup plus profitable de se ménager dès le début des relations cordiales avec le syndicat de ses employés.

OMER - Genest 


\section{FAUT-IL PREVENIR LE PATRON}

\section{Organisation syndicale}

Des employés décident de former un syndicat; ils prennent contact avec un agent d'affaires au service d'une organisation ouvrière pour obtenir son concours. Doivent-ils aussitôt rencontrer leur patron pour le mettre au courant de leur intention de se grouper? ou l'agent d'affaires doit-il se charger de cette tâche? S'ils ne le font pas, peuvent-ils être accusés de travailler dans le dos du patron ?

Pour répondre à ces questions, il faut tenir compte de l'état d'esprit des personnes en cause et des circonstances où elles agissent.

Des ouvriers qui songent à s'organiser sont toujours craintifs : ils se demandent comment leur patron va réagir à l'annonce de cette nouvelle. Ils ont beau se savoir protégés par la loi qui garantit leur liberté syndicale, ils n'ignorent pas que ses violateurs sont difficilement poursuivis et mis à la raison; les ouvriers congédiés pour activité unioniste dans les premiers temps d'un syndicat risquent de n'être guère soutenus par leurs confrères, qui n'ont pas encore appris que la solidarité constitue la seule force des faibles. C'est pourquoi au début d'un syndicat, les ouvriers ont peur de se mettre de l'avant; dans leur incertitude timorée, aucun d'eux n'osera se compromettre et affronter le patron.

L'agent d'affaires, qui est indépendant de l'employeur, ne redoute aucune représaille contre lui. Mais s'il intervient, il risque de se faire traiter d'《outsider», qui ne devrait pas se mêler de ce qui ne le regarde pas. Dans le fond, le patron est vexé de constater que ses employés ont été trop timides pour communiquer avec lui eux-mêmes et il voit d'un mauvais oeil cet étranger qui va acquérir un certain ascendant sur ses employés et avec qui il ne voudrait pas avoir à traiter.

Bref, si les ouvriers préfèrent attendre que leur syndicat soit bien établi pour entrer en communication avec le patron, c'est qu'ils redoutent une réaction défavorable de sa part. Le point est de savoir si ces craintes sont fondées.

I1 se rencontre des patrons prêts à accueillir avec bienveillance l'union commençante de leurs employés. Mais ils sont excessivement rares et constituent une minime exception.

La plupart de ceux qui affirment hautement leur appui au syndicalisme et déclarent n'avoir aucune objection à ce que leurs employés s'organisent, n'en combattent pas moins en pratique le syndicat nouveau sous prétexte que tel officier ou tel membre influent est une mauvaise tête, que les plus anciens employés n'en font pas partie, que l'agent d'affaires ou le conseiller technique qui le dirige est un agitateur ou un homme intraitable. Ils reprochent au syndicat de pousser les esprits au mécontentement et de briser les relations cordiales qu'ils entretenaient avec leurs employés en leur inspirant des exigences extravagantes. Etudions ces objections.

Que les unions ouvrières doivent se préoccuper d'avoir de bons chefs et des porte-paroles acceptables, entendu. Mais ce n'est pas au patron à choisir les dirigeants ou les agents négociateurs de ses employés; pas plus que ceux-ci ont à déterminer le gérant avec qui ils traitent. $\mathrm{La}$ plus grande erreur stratégique que les uns ou les autres puissent commettre, c'est d'échapper en quelque occasion des paroles désobligeantes contre les représentants de l'autre partie. De part et d'autre, en dépit des préjugés ou des défauts, les hommes au poste, quels qu'ils soient, n'ont qu'à essayer de s'entendre pour le mieux. Avec une bonne volonté, humble et loyale, on y réussit toujours.

Que, lors des premières réunions du syndicat, des critiques manifestent un mécontentement plus ou moins profond, l'employeur en l'apprenant par des bavards indiscrets a tort de s'en prendre au syndicat. S'il est un réaliste, il comprendra que l'union donne simplement l'occasion à ses hommes de dire tout haut ce qu'ils pensent en eux-mêmes. Or, l'employé fournit son plein rendement seulement s'il travaille avec un bon moral. Le patron, dont l'orgueil n'obnubile pas le sens des affaires, sait donc qu'il a intérêt à connaitre les griefs de ses employés. Lorsqu'ils sont fondés, il s'empressera d'en corriger les causes; autrement, il verra à mieux informer son personnel pour lui faire comprendre ce qui justifie telle mesure, telle décision qui a indisposé les esprits. Le syndicat lui sera ici d'un précieux secours. Non, le syndicat ne crée pas le mécontentement; il donne l'opportunité d'exprimer des' critiques qui couvaient dans les esprits et il est le moyen irremplaçable de régler bien des griefs déprimants.

L'union va transformer les relations du patron avec ses employés. Il a traité avec eux individuellement, d'une façon très paternelle sans doute, mais tout de même beaucoup à sa guise et sans avoir à expliquer ses décisions. Les employés isolés acceptaient tout de la libéralité du patron ou subissaient, impuissants, des mesures qu'ils jugaient arbitraires ou injustes.

(Suite à la page 7) 